\title{
Ten years in two dimensions
}

\author{
Are graphene technologies ready for commercialization?
}

It is ten years since Andre Geim and Kostya Novoselov published their seminal work on the physics of graphene ${ }^{1}$ and research into this two-dimensional material has progressed quickly in that time. Scientists have revealed a range of intriguing properties and have highlighted the potential of graphene in a variety of important applications, from electronics to energy storage. These findings have led to the search for other two-dimensional materials. Advances in synthesis have allowed larger and larger quantities of graphene to be grown and for an increasing number of researchers to have access to sufficient quantities. A Nobel Prize has been awarded, and massive public investment has been provided. (The European Commission has, for example, recently financed a 10-year research initiative the European Graphene Flagship - that will provide up to $€ 1$ billion in funding and involves more than 140 academic and commercial institutions in 23 countries $^{2}$.) Industry has also taken note and is now exploring a range of potential applications. But how close are we to seeing graphene deliver the profound applications that have been proposed? In this issue of Nature Nanotechnology, in a focus on graphene applications, we provide a snapshot of the current status of key graphene technologies.

The introduction of graphene-based devices in industrial applications will require large-area graphene films, which are usually polycrystalline and therefore do not necessarily have the same properties as pristine, single-crystal films. As Oleg Yazyev and Yong Chen explain in the first of four Review articles, the mechanical, thermal, optical and electronic properties of polycrystalline graphene are sensitively dependent on grain boundaries and other defects $^{3}$. The optimization of large-scale growth methods will therefore require a thorough understanding of microscopic structural processes, and an ability to engineer them. This will also need to be complemented by post-growth treatments to achieve the desired macroscopic properties.

One of the most striking features of graphene is its high charge-carrier mobility, and electronic and optoelectronic applications that could exploit this, as well as other characteristics, were among the first to be envisaged for the material. In our second Review, Francesco Bonaccorso and colleagues

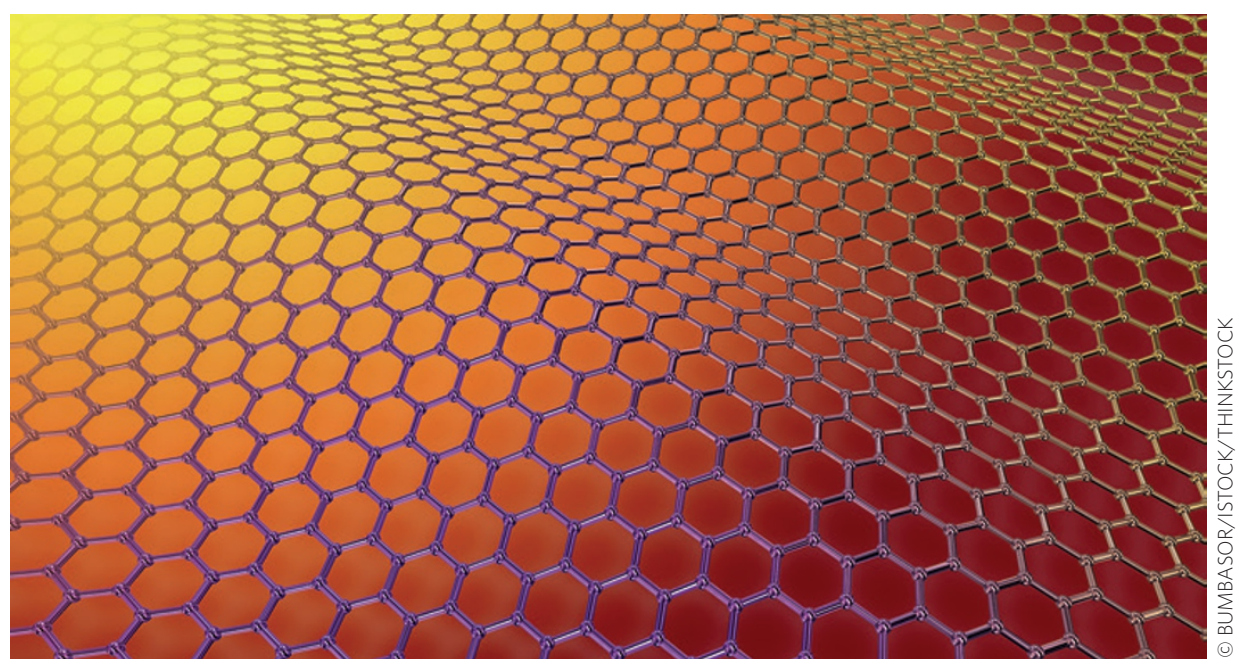

discuss developments in electronics based on graphene and related layered materials, which could potentially replace silicon-based electronic components in both digital and analog applications by leveraging on the ultimate thickness of these materials and on their excellent mobility ${ }^{4}$. Light-matter interactions in graphene can lead to sensitive and fast light detection in a spectral range from ultraviolet to terahertz frequencies, and the graphene photoresponse can be electrically tuned by gating; Frank Koppens and colleagues explore these properties in our third Review article 5 . Then in our final Review, Roland Kawakami and colleagues examine the properties of graphene that are relevant to spintronics ${ }^{6}$. The material is of interest in spintronics because of its predicted long spin lifetimes, and although progress has been made to achieve spin-injection in graphene, efforts are still being devoted to fully understand spin-relaxation mechanisms.

The outstanding properties of graphene also make it a suitable material for an assortment of other applications. To explore this range, we asked seven experts to discuss the prospects and challenges for the commercialization of graphene-based products in a number of key areas ${ }^{7}$. While these graphene applications are each at different levels of maturity, and each face different challenges, a recurrent theme in these contributions is the need to achieve large-scale production of graphene materials.

In the first of two Commentary articles, Wencai Ren and Hui-Ming Cheng discuss the worldwide industrial production capacity of both small graphene sheets and large-area graphene films ${ }^{8}$. They highlight the special role that China can play in supplying graphene materials, due to its rapidly developing manufacturing industry. However, high-volume production is only one challenge towards commercialization. In our second Commentary, Amaia Zurutuza and Claudio Marinelli provide an overview of factors that may either slow down or speed up the adoption of graphene in industrial applications. They indicate that high manufacturing costs, resistance from existing technologies, and health and safety concerns are, in particular, important issues to address; on the other hand, they suggest that the identification of markets with low barriers-toentry could accelerate the widespread uptake of graphene products.

A decade on from the work of Geim and Novoselov, only a handful of graphene-based products are available off the shelf. But as our focus on graphene applications makes clear, the next few years are likely to see the emergence of a number of significant commercial opportunities for graphene and other two-dimensional materials.

\footnotetext{
References

1. Novoselov, K. S. et al. Science 306, 666-669 (2004).

2. http://graphene-flagship.eu/wp-content/uploads/2014/09/Press1Release_Graphene-Flagship-Competitive-Call-GF-.pdf

. Yazyev, O. V. \& Chen, Y. P. Nature Nanotech. 9, 755-767 (2014)

4. Fiori, G. et al. Nature Nanotech. 9, 768-779 (2014).

5. Koppens, F. H. L. et al. Nature Nanotech. 9, 780-793 (2014).

6. Han, W., Kawakami, R. K., Gmitra, M. \& Fabian, J.

Nature Nanotech. 9, 794-807 (2014).

Nature Nanotech. 9, 737-747 (2014).

8. Ren, W. \& Cheng, H.-M. Nature Nanotech. 9, 726-730 (2014).

9. Zurutuza, A. \& Marinelli, C. Nature Nanotech. 9, 730-734 (2014)
} 